\title{
Conductance from Non-perturbative Methods II
}

\section{Olalla A. Castro-Alvaredo* and Andreas Fring}

Institut für Theoretische Physik, Freie Universität Berlin, Arnimallee 14, D-14195 Berlin, Germany

E-mail: 'olalla@physik.fu-berlin.de', Fring@physik.fu-berlin.dé,

ABstract: This talk provides a natural continuation of the talk presented by Andreas Fring in this conference. Part I was focused on explaining how the DC conductance for a free Fermion theory in the presence of different kinds of defects can be computed by evaluating the Kubo formula. In this talk I will focus on an alternative method for the computation of the same quantity, that is the evaluation of Landauer formula. Once again, the integrability of the theories under consideration will be exploited, since a thermodynamic Bethe ansatz analysis provides all the input needed in that case, apart from the corresponding reflection and transmition amplitudes of the defect. The basic conclusion of our analysis will be the perfect agreement between the two different theoretical descriptions mentioned.

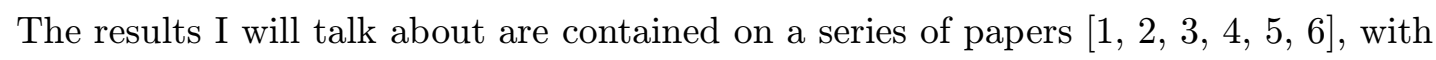
emphasis on the first two, resulting mainly from a collaboration with Andreas Fring, who presented the first part of the work.

\section{Thermodynamic Bethe ansatz for impurity systems}

As mentioned in the previous talk, for the evaluation of the Landauer formula $\left[\begin{array}{l}{[\overline{2}} \\ ]\end{array}\right]$ one needs to know the density distribution functions involved. I will now present a general method which allows to compute such quantities non-perturbatively, i.e. the thermodynamic Bethe ansatz (TBA) approach, which we generalized in [i] $\left[\begin{array}{l}1 \\ 1\end{array}\right]$ to incorporate the non-trivial effects arising due to the presence of impurities. Besides the aim we have in mind, in general the TBA is a powerful tool for the computation of thermodynamic quantities in $1+1$ dimensional integrable systems. Originally formulated by Yang and Yang [isi] in the context of the non-relativistic Bose gas, it was thereafter generalized by Zamolodchikov [ịg] to relativistic quantum field theories which interact by means of factorizable scattering matrices. A TBA-analysis serves to check the consistency of a certain S-matrix proposal, since it allows

\footnotetext{
${ }^{*}$ Speaker.
} 
for extracting some distinct structural quantities such as the Virasoro central charge of the underlying conformal field theory. The original bulk formulation has been accommodated to a situation which includes a purely transmitting defect in [i] $\left.\overline{0}_{1}^{0}\right]$, whereas for purely reflecting impurities (that is, boundaries) the TBA equations were newly derived in [i] $\left.\overline{1}_{1}^{1}\right]$. In this section we want to propose a new formulation which, for the first time, will hold for a situation when non-vanishing reflection and transmission occur simultaneously. Let us consider first of all the standard starting point in the formulation of the Bethe ansatz equations: We consider a $1+1$ dimensional system with compactified space dimension $L$ and $N$ particles distributed as shown in figure 1. As standard in this context, we represent those particles by means of the Zamolod- chikov-Faddeev (ZF) operators [1' $1 \overline{2}] Z_{i}(\theta)$. In addition,

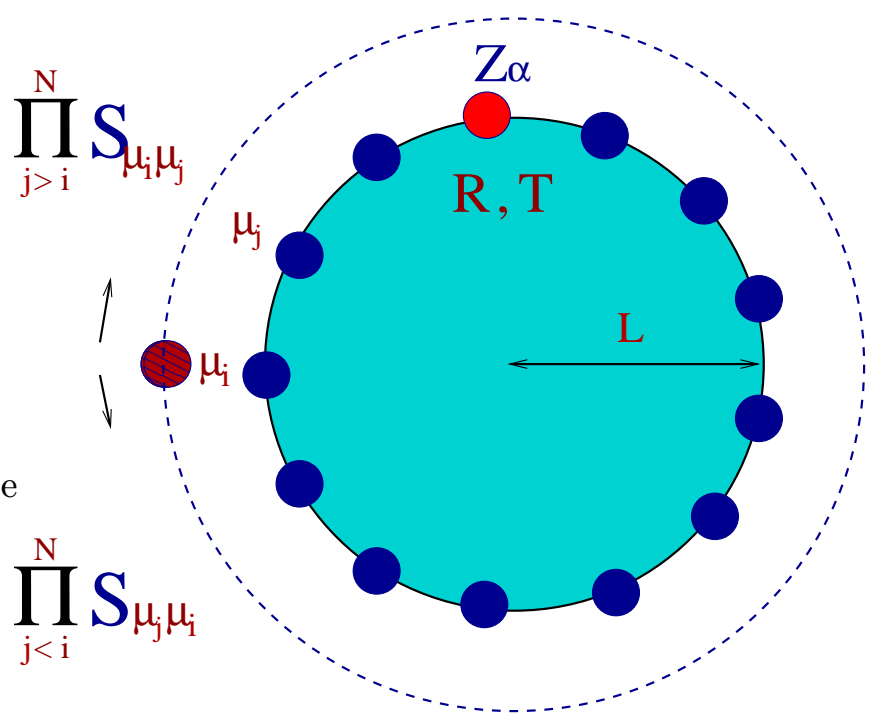

Figure 1: The Bethe wave function for a defect system. in order to incorporate the pres-

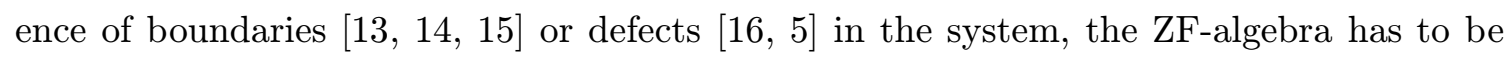
extended with new generators $Z_{\alpha}$. We indicate particle types by Latin and degrees of freedom of the impurity by Greek letters. The "braiding" (exchange) relations of annihilation operators $Z_{i}(\theta)$ of a particle of type $i$ moving with rapidity $\theta$ and defect operators $Z_{\alpha}$ in the state $\alpha$ can be written as

$$
\begin{aligned}
Z_{i}\left(\theta_{1}\right) Z_{j}\left(\theta_{2}\right) & =S_{i j}^{k l}\left(\theta_{1}-\theta_{2}\right) Z_{k}\left(\theta_{2}\right) Z_{l}\left(\theta_{1}\right) \\
Z_{i}\left(\theta_{1}\right) Z_{j}^{\dagger}\left(\theta_{2}\right) & =S_{i j}^{k l}\left(\theta_{1}-\theta_{2}\right) Z_{k}^{\dagger}\left(\theta_{2}\right) Z_{l}\left(\theta_{1}\right)+2 \pi \delta\left(\theta_{1}-\theta_{2}\right) \delta_{i j}, \\
Z_{i}(\theta) Z_{\alpha} & =R_{i \alpha}^{j \beta}(\theta) Z_{j}(-\theta) Z_{\beta}+T_{i \alpha}^{j \beta}(\theta) Z_{\beta} Z_{j}(\theta) \\
Z_{\alpha} Z_{i}(\theta) & =\tilde{R}_{i \alpha}^{j \beta}(-\theta) Z_{\beta} Z_{j}(-\theta)+\tilde{T}_{i \alpha}^{j \beta}(-\theta) Z_{j}(\theta) Z_{\beta} .
\end{aligned}
$$

The bulk scattering matrix is indicated by $S$, and the left/right reflection and transmission amplitudes through the defect are denoted by $R / \tilde{R}$ and $T / \tilde{T}$, respectively as seen in part I. We employed Einstein's sum convention, that is we assume sums over doubly occurring indices. We suppress the explicit mentioning of the dependence of $Z_{\alpha}$ on the position in space and assume for the time being that it is included in $\alpha$. For the treatment of a single defect this is not relevant, but it will become once more important when we consider multiple defects. The same relations hold when we replace the annihilation operators by the creation operators $Z_{i}^{\dagger}(\theta)$ with $R / \tilde{R}, T / \tilde{T}$ and $S$ replaced by their complex conjugates. The algebra (1) (2.1; part I) and (2.2; part I) which result just from applying it twice. As usual, we obtain the Bethe ansatz equations by dragging a particle $i$ along the world line. We introduce for 
convenience the following shorthand notation for the product of various particle operators $Z_{i}(\theta)$ and a defect operator $Z_{\alpha}$

$$
Z_{k, \alpha}^{\mu_{1} \ldots \mu_{N}}:=Z_{\mu_{1}}\left(\theta_{\mu_{1}}\right) \ldots Z_{\mu_{k}}\left(\theta_{\mu_{k}}\right) Z_{\alpha} Z_{\mu_{k+1}}\left(\theta_{\mu_{k}+1}\right) \ldots Z_{\mu_{N}}\left(\theta_{\mu_{N}}\right) .
$$

Then we compute the braiding of a particle operator of type $i$ and the previous product

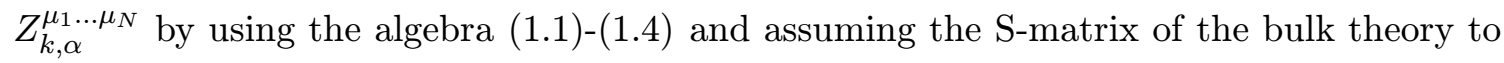
be diagonal

$$
\begin{aligned}
& Z_{i}\left(\theta_{i}\right) Z_{k, \alpha}^{\mu_{1} \ldots \mu_{N}}=Z_{k, \alpha}^{\mu_{1} \ldots \mu_{N}} Z_{i}\left(\theta_{i}\right) \tilde{F}_{i \alpha}-Z_{k, \alpha}^{\mu_{1} \ldots \mu_{N}} Z_{i}\left(-\theta_{i}\right) \tilde{G}_{i \alpha}, \\
& Z_{k, \alpha}^{\mu_{1} \ldots \mu_{N}} Z_{i}\left(\theta_{i}\right)=Z_{i}\left(\theta_{i}\right) Z_{k, \alpha}^{\mu_{1} \ldots \mu_{N}} F_{i \alpha}-Z_{i}\left(-\theta_{i}\right) Z_{k, \alpha}^{\mu_{1} \ldots \mu_{N}} G_{i \alpha} .
\end{aligned}
$$

We abbreviated here

$$
\begin{array}{ll}
\tilde{F}_{i}^{\alpha}=\frac{1}{\tilde{T}_{i}^{\alpha}\left(-\theta_{i}\right)} \prod_{l=1}^{N} S_{i \mu_{l}}\left(\theta_{i \mu_{l}}\right), & \tilde{G}_{i}^{\alpha}=\frac{\tilde{R}_{i}^{\alpha}\left(-\theta_{i}\right)}{\tilde{T}_{i}^{\alpha}\left(-\theta_{i}\right)} \prod_{l=1}^{k} S_{i \mu_{l}}\left(\theta_{i \mu_{l}}\right) \prod_{l=k+1}^{N} S_{i \mu_{l}}\left(-\hat{\theta}_{i \mu_{l}}\right), \\
F_{i}^{\alpha}=\frac{1}{T_{i}^{\alpha}\left(\theta_{i}\right)} \prod_{l=1}^{N} S_{\mu_{l} i}\left(\theta_{\mu_{l} i}\right), & G_{i}^{\alpha}=\frac{R_{i}^{\alpha}\left(\theta_{i}\right)}{T_{i}^{\alpha}\left(\theta_{i}\right)} \prod_{l=1}^{k} S_{\mu_{l} i}\left(\hat{\theta}_{\mu_{l} i}\right) \prod_{l=k+1}^{N} S_{\mu_{l} i}\left(\theta_{\mu_{l} i}\right) .
\end{array}
$$

Being on a circle of length $L$, we can make the usual assumption on the Bethe wavefunction (see e.g. [igi]) which is captured in the requirement

$$
Z_{i}(\theta) Z_{k, \alpha}^{\mu_{1} \ldots \mu_{N}}=Z_{k, \alpha}^{\mu_{1} \ldots \mu_{N}} Z_{i}(\theta) \exp \left(-i L m_{i} \sinh \theta\right) .
$$

Using this monodromy property together with the braiding relations ( unitarity relations for $R$ and $T$ (see section 2 of part I), we obtain the following Bethe ansatz equations

$$
\prod_{l=1}^{N} \frac{S_{l i}\left(\hat{\theta}_{l i}\right)}{S_{l i}\left(\theta_{l i}\right)}\left(\prod_{l=1}^{N} S_{l i}\left(\theta_{l i}\right)-\frac{e^{i L m_{i} \sinh \theta_{i}}}{\tilde{T}_{i}^{\alpha}\left(-\theta_{i}\right)}\right)=\frac{T_{i}^{\alpha}\left(-\theta_{i}\right)}{\tilde{T}_{i}^{\alpha}\left(-\theta_{i}\right)}\left(\frac{e^{-i L m_{i} \sinh \theta_{i}}}{T_{i}^{\alpha}\left(\theta_{i}\right)}-\prod_{l=1}^{N} S_{i l}\left(\theta_{i l}\right)\right) .
$$

We restrict it here to the diagonal case, i.e. $S_{i j}^{k l}(\theta)=S_{i j}(\theta) \delta_{l i} \delta_{k j}, R_{i \alpha}^{j \beta}(\theta)=R_{i}^{\alpha}(\theta) \delta_{\alpha \beta} \delta_{i j}$, $T_{i \alpha}^{j \beta}(\theta)=T_{i}^{\alpha}(\theta) \delta_{\alpha \beta} \delta_{i j}$ and similarly for the tilde amplitudes. We can therefore use the result mentioned in part I, namely that for $R$ and $T$ to be simultaneously non-vanishing the only possible bulk scattering matrices are $S= \pm 1$, such that the relation (1.11) may be re-written as

$$
1=e^{i L m_{i} \sinh \theta} D_{i \alpha}^{ \pm}(\theta) \prod_{l=1}^{N} S_{i l}
$$

where

$$
D_{i \alpha}^{ \pm}(\theta)=\frac{\tilde{T}_{i}^{\alpha}(\theta)+T_{i}^{\alpha}(\theta) \prod_{l=1}^{N} S_{i l}^{2}}{2} \pm \frac{1}{2}\left[\left(\tilde{T}_{i}^{\alpha}(\theta)+T_{i}^{\alpha}(\theta) \prod_{l=1}^{N} S_{i l}^{2}\right)^{2}-\frac{4 T_{i}^{\alpha}(\theta) \prod_{l=1}^{N} S_{i l}^{2}}{T_{i}^{\alpha}(-\theta)}\right]^{\frac{1}{2}}
$$

For consistency reasons it is instructive to consider the limit when the reflection amplitude tends to zero. In that case we can employ the unitarity relations for the reflection and 
transmission amplitudes (see section 2 of part I) and may take the square root in (11.13), such that we obtain from (1.12i) the two equations

$$
R, \tilde{R} \rightarrow 0: \quad 1=e^{i L m_{i} \sinh \theta} \tilde{T}_{i}^{\alpha}(\theta) \prod_{l=1}^{N} S_{i l}, \quad 1=e^{-i L m_{i} \sinh \theta} T_{i}^{\alpha}(\theta) \prod_{l=1}^{N} S_{l i} .
$$

This means we recover the Bethe ansatz equations for a purely transmitting defect, which were originally proposed by Martins in [1] of parity invariance in the limiting case, i.e. the two equations in (1.14) correspond to taking the particle either clockwise or anti-clockwise around the world line as formulated for the parity breaking case for the first time in [i] $\left.{ }_{1}^{1} \bar{i}\right]$ and explicitly indicated in figure 1 . We do not expect to recover from here the equations for a purely reflecting boundary which

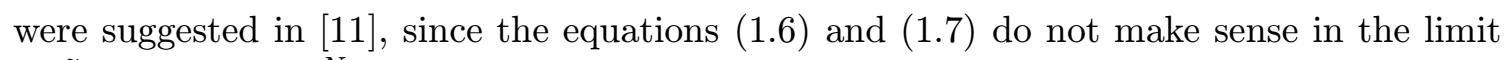
$T, \tilde{T} \rightarrow 0$. For $\prod_{l=1}^{N^{-}} S_{i l}^{2}=1$, i.e. the free Boson and Fermion, we can exploit the fact that $(1 . \overline{1} 2)$ with (1.13i) look formally precisely like the Bethe ansatz equations for a purely transmitting defect. If we want to exploit this analogy we should of course be concerned about the question whether $D_{j \alpha}^{ \pm}(\theta)$ is a meromorphic function. Assuming parity invariance, we may take the square root

$$
D_{j \alpha}^{ \pm}(\theta)=T_{j}^{\alpha}(\theta) \pm R_{j}^{\alpha}(\theta) \quad \text { for } \quad R=\tilde{R}, T=\tilde{T} .
$$

The matrix $D_{j \alpha}^{ \pm}(\theta)$ has now the usual properties, namely it is unitarity in the sense that $D_{j \alpha}^{ \pm}(\theta) D_{j \alpha}^{ \pm}(-\theta)=1$. It follows further from (1.15i) and from the crossing relations for $R$ and $T$ that the hermiticity relation $D_{j \alpha}^{ \pm}(\theta)=D_{j \alpha}^{ \pm}(-\theta)^{*}$ and the crossing relations $D_{\bar{\jmath} \alpha}^{ \pm}(\theta)=$ $D_{j \alpha}^{\mp}(i \pi-\theta)$ and $D_{\bar{j} \alpha}^{ \pm}(\theta)=D_{j \alpha}^{ \pm}(i \pi-\theta)$ hold for the free Fermion and Bosons, respectively.

Let us now carry out the thermodynamic limit in the usual way, namely by increasing the particle number $N$ and the system size $L$ in such a way that their mutual ratio $N / L$ remains finite. The amount of defects will be kept constant in this limit, such that there is no contribution to the TBA-equations from the defect in that situation. The same behaviour was pointed out in [1] $\left.{ }_{1}^{1} \overline{0}\right]$ for the purely transmitting case. Intuitively the latter result was to be expected, since making both the amount of particles and the size of the system infinite while keeping the amount of defects fixed will lead to a situation in which the effect of the presence of a finite number of defects is negligeable. Hence, this means that essentially we can employ the usual bulk TBA analysis when the considerations are carried out in the thermodynamic limit.

Let us therefore recall the main equations of the TBA analysis. For more details on the derivation see $[\overline{9} \overline{9}]$ and in particular for the introduction of the chemical potential see [i] 1 in]. The main input into the entire analysis is the dynamical interaction, which enters via the logarithmic derivative of the scattering matrix $\varphi_{i j}(\theta)=-i d \ln S_{i j}(\theta) / d \theta$ and the

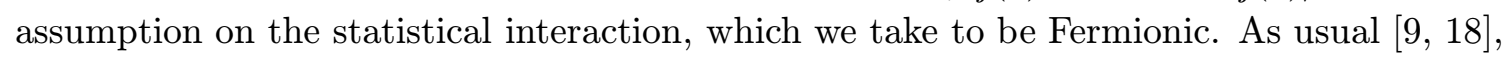
we take the logarithmic derivative of the Bethe ansatz equation (1; $\left.12_{1}^{\prime}\right)$ and relate the density of states $\rho_{i}(\theta, r)$ for particles of type $i$ as a function of the inverse temperature $r=1 / T$ to the density of occupied states $\rho_{i}^{r}(\theta, r)$

$$
\rho_{i}(\theta, r)=\frac{m_{i}}{2 \pi} \cosh \theta+\sum_{j}\left[\varphi_{i j} * \rho_{i}^{r}\right](\theta)
$$


By $(f * g)(\theta):=1 /(2 \pi) \int d \theta^{\prime} f\left(\theta-\theta^{\prime}\right) g\left(\theta^{\prime}\right)$ we denote as usual the convolution of two functions. The mutual ratio of the densities serves as the definition of the so-called pseudoenergies $\varepsilon_{i}(\theta, r)$

$$
\frac{\rho_{i}^{r}(\theta, r)}{\rho_{i}(\theta, r)}=\frac{e^{-\varepsilon_{i}(\theta, r)}}{1+e^{-\varepsilon_{i}(\theta, r)}},
$$

which have to be positive and real. Notice that, from the introduction in part I, the quantities $\rho_{i}^{r}(\theta, r)$ at the constrictions of the wire are the basic input we need for the evaluation of Landauer formula, apart from the reflection and transmission amplitudes. At thermodynamic equilibrium one obtains then the TBA-equations, which read in these variables and in the presence of a chemical potential $\mu_{i}$

$$
r m_{i} \cosh \theta=\varepsilon_{i}\left(\theta, r, \mu_{i}\right)+r \mu_{i}+\sum_{j}\left[\varphi_{i j} * \ln \left(1+e^{-\varepsilon_{j}}\right)\right](\theta),
$$

where $r=m / T, m_{l} \rightarrow m_{l} / m, \mu_{i} \rightarrow \mu_{i} / m$, with $m$ being the mass of the lightest particle in the model. It is important to note that $\mu_{i}$ is restricted to be smaller than 1 . This follows immediately from (1) $\left.\overline{1}_{1} \overline{1}_{i}^{\prime}\right)$ by recalling that $\varepsilon_{i} \geq 0$ and that for $r$ large $\varepsilon_{i}\left(\theta, r, \mu_{i}\right)$ tends to infinity. As pointed out already in [9in] (here just with the small modification of a chemical

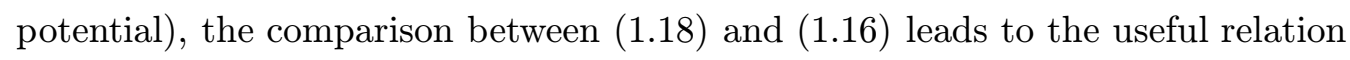

$$
\rho_{i}\left(\theta, r, \mu_{i}\right)=\frac{1}{2 \pi}\left(\frac{d \varepsilon_{i}\left(\theta, r, \mu_{i}\right)}{d r}+\mu_{i}\right) .
$$

The main task is therefore first to solve (1.18) for the pseudo-energies from which then all densities can be reconstructed.

\subsection{Thermodynamic quantities per unit length}

Treating the equations (1.12i) and (1.13i) in the mentioned analogy with the purely transmitting case we can also construct various thermodynamic quantities. It should be stressed that these quantities are computed per unit length. Similarly as the expression found in [1] $[10 \overline{0}]$ for a purely transmitting defect the free energy is

$$
F(r)=-\frac{1}{\pi r} \sum_{l, \alpha} \hat{m}_{l} \int_{0}^{\infty} d \theta\left[\cosh \theta+m^{-1} \varphi_{l \alpha}(\theta)\right] \ln [1+\exp (-r m \cosh \theta)] .
$$

It is made up of two parts, one coming from the bulk and one including the data of the defect in form of $\varphi_{l \alpha}(\theta)=-i d \ln D_{l \alpha}(\theta) / d \theta$. From equation (1.20i) we also see that when taking the mass scale to be large in comparison to the dominating scale in the defect, the latter contribution to the scaling function becomes negligible with regard to the bulk and vice versa. However, in this talk we shall concentrate on the thermodynamic limit.

\section{Conductance through an impurity}

The most intuitive way to compute the conductance is via Landauer-Büttinger transport theory [i] middle of a rigid bulk wire, where the two halves might be at different temperatures. The 
direct current $I$ through such a quantum wire can be computed simply by determining the difference between the static charge distributions at the right and left constriction of the

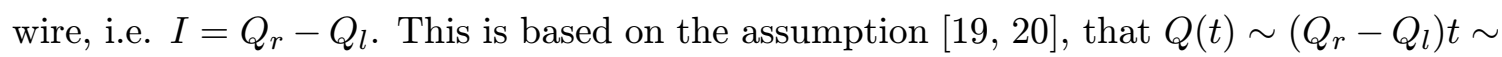
$\left(\rho_{r}-\rho_{l}\right) t$, where the $\rho$ s are the corresponding density distribution functions. Placing an

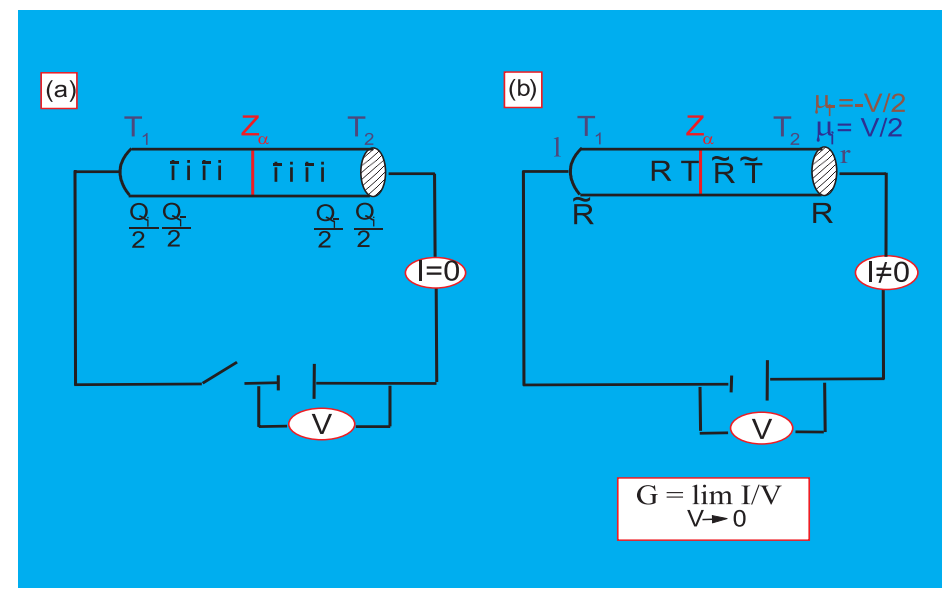

Figure 2: A conductance measurement. Part (a) represents the initial condition with no current flowing, i.e., $\mathrm{I}=0$ and part (b), $I \neq 0$. The defect is placed in the middle of the wire and the left and right half are assumed to be at temperatures $T_{1}$ and $T_{2}$, respectively.

impurity in the middle of the wire, we have to quantify the overall balance of particles of type $i$ and anti-particles $\bar{\imath}$ carrying opposite charges $q_{i}=-q_{\bar{\imath}}$ at the end of the wire at different potentials. This information is of course encoded in the density distribution function $\rho_{i}^{r}\left(\theta, T, \mu_{i}\right)$. In the described set up half of the particles of one type are already at the same potential at one of the ends of the wire and the probability for them to reach the other is determined by the transmission and reflection amplitudes through the impurity. We assume that there is no effect coming from the constrictions of the wire, i.e. they are purely transmitting surfaces with $T=\tilde{T}=1$. One could, however, also consider a situation in which those constrictions act as boundaries, namely purely reflecting surfaces. The situation could be described with the same transport theory picture, see e.g. [1] $\left.{ }_{1}^{1} \overline{9}_{1}^{1}, 2 \overline{1} \overline{1}_{1}^{1}, \overline{2}_{2} \overline{2} \overline{1}\right]$, but then the conductance can only be non-vanishing if the reflection amplitudes in the constrictions are non-diagonal in the particle degrees of freedom, such as for instance for sine-Gordon [23], that is in general affine Toda field theories with purely imaginary coupling constant or, in the massless limit, folded purely reflecting (transmitting) diagonal bulk theories.

According to the Landauer transport theory the direct current (DC) along the wire is given by

$$
I^{\vec{\alpha}}=\sum_{i} I_{i}^{\vec{\alpha}}\left(r, \mu_{i}^{l}, \mu_{i}^{r}\right)=\sum_{i} \frac{q_{i}}{2} \int_{-\infty}^{\infty} d \theta\left[\rho_{i}^{r}\left(\theta, r, \mu_{i}^{r}\right)\left|T_{i}^{\vec{\alpha}}(\theta)\right|^{2}-\rho_{i}^{r}\left(\theta, r, \mu_{i}^{l}\right)\left|\tilde{T}_{i}^{\vec{\alpha}}(\theta)\right|^{2}\right],
$$




$$
=I_{B}-\sum_{i} \frac{q_{i}}{2} \int_{-\infty}^{\infty} d \theta\left[\rho_{i}^{r}\left(\theta, r, \mu_{i}^{r}\right)\left|R_{i}^{\vec{\alpha}}(\theta)\right|^{2}-\rho_{i}^{r}\left(\theta, r, \mu_{i}^{l}\right)\left|\tilde{R}_{i}^{\vec{\alpha}}(\theta)\right|^{2}\right],
$$

where we assume here $T_{1}=T_{2}$. The relation $\left(\overline{2} \cdot \overline{2}_{1}^{1}\right)$ is obtained from $\left(\overline{2} \cdot \overline{1}_{1}\right)$ simply by making use of the fact that $|R|^{2}+|T|^{2}=1$ (see section 2 in part I). Equation (2.2) has the virtue that it extracts explicitly the bulk contribution to the current which we refer to as $I_{B}$. There are some obvious limits, namely a transparent and an impenetrable defect

$$
\lim _{\left|T^{\vec{\alpha}}\right| \rightarrow 1} I^{\vec{\alpha}}=I_{B} \quad \text { and } \quad \lim _{\left|T^{\vec{\alpha}}\right| \rightarrow 0} I^{\vec{\alpha}}=0
$$

respectively. A short comment is needed on the validity of (2.1). Apparently it suggests that when the parity between left and right scattering is broken, there is the possibility of a net current even when an external source is absent. In this picture we have of course not taken into account that charged particles moving through the defect will alter the potential, such that we did in fact not describe a perpetuum mobile. Thus the limitation of our analysis is that $\mu_{i}^{l}-\mu_{i}^{r}$ has to be much larger than the change in the potential induced by the moving particles.

Finally we want to compute the conductance from the DC current, which by definition is obtained from

$$
G^{\vec{\alpha}}(r)=\sum_{i} G_{i}^{\vec{\alpha}}(r)=\sum_{i\left(\mu_{i}^{l}-\mu_{i}^{r}\right) \rightarrow 0} I_{i}^{\vec{\alpha}}\left(r, \mu_{i}^{l}, \mu_{i}^{r}\right) /\left(\mu_{i}^{l}-\mu_{i}^{r}\right)
$$

and is of course a property of the material itself and a function of the temperature. In general the expressions in $\left(\overline{2}_{2}^{2} . \bar{l}_{1}\right)$ tend to zero for vanishing chemical potential difference such that the limit in $(\overline{2}-\overline{4})$ is non-trivial.

Thus from the knowledge of the transmission matrix and the density distribution function we can compute the conductance.

\subsection{The high temperature regime}

Since the physical quantities require a solution of the TBA-equations, which up to now, due to their non-linear nature, can only be solved numerically, we have to resort in general to a numerical analysis to obtain the conductance for some concrete theories. However, there exist various approximations for different special situations, such as the high temperature regime. For large rapidities and small $r$, it is known [9.9-1] (here we only need the small modification of the introduction of a chemical potential $\mu_{i}$ ) that the density of states can be approximated by

$$
\rho_{i}\left(\theta, r, \mu_{i}\right) \sim \frac{m_{i}}{4 \pi} e^{|\theta|} \sim \frac{1}{2 \pi r} \epsilon(\theta) \frac{d \varepsilon_{i}\left(\theta, r, \mu_{i}\right)}{d \theta},
$$

where $\epsilon(\theta)=\Theta(\theta)-\Theta(-\theta)$ is the step function, i.e. $\epsilon(\theta)=1$ for $\theta>0$ and $\epsilon(\theta)=-1$ for $\theta<0$. In equation (1.1), we assume that in the large rapidity regime $\rho_{i}^{r}\left(\theta, r, \mu_{i}\right)$ is dominated by $(\overline{2} . \overline{2} .5)$ and in the small rapidity regime by the Fermi distribution function. Therefore

$$
\rho_{i}^{r}\left(\theta, r, \mu_{i}\right) \sim \frac{1}{2 \pi r} \epsilon(\theta) \frac{d}{d \theta} \ln \left[1+\exp \left(-\varepsilon_{i}\left(\theta, r, \mu_{i}\right)\right)\right] .
$$


Using this expression in equation $(2 . \overline{2} \cdot \overline{1})$, we approximate the direct current in the ultraviolet by

$$
\lim _{r \rightarrow 0} I_{i}^{\vec{\alpha}}\left(r, \mu_{i}\right) \sim \frac{q_{i}}{4 \pi r} \int_{-\infty}^{\infty} d \theta \ln \left[\frac{1+\exp \left(-\varepsilon_{i}\left(\theta, r, \mu_{i}^{l}\right)\right)}{1+\exp \left(-\varepsilon_{i}\left(\theta, r, \mu_{i}^{r}\right)\right)}\right] \frac{d\left[\epsilon(\theta)\left|T_{i}^{\vec{\alpha}}(\theta)\right|^{2}\right]}{d \theta},
$$

after a partial integration. For simplicity we also assumed here parity invariance, that is $\left|T_{i}^{\alpha}(\theta)\right|=\left|\tilde{T}_{i}^{\alpha}(\theta)\right|$. The derivation of the analogue to $\left(\overline{2}_{2} . \bar{T}_{i}\right)$ for the situation when parity is broken is of course similar. Taking now the potentials at the end of the wire to be $\mu_{i}^{r}=-\mu_{i}^{l}=V / 2$, the conductance reads in this approximation

$$
\left.\lim _{r \rightarrow 0} G_{i}^{\vec{\alpha}}(r) \sim \frac{q_{i}}{2 \pi r} \int_{-\infty}^{\infty} d \theta \frac{1}{1+\exp \left[\varepsilon_{i}(\theta, r, 0)\right]} \frac{d \varepsilon_{i}(\theta, r, V / 2)}{d V}\right|_{V=0} \frac{d\left[\epsilon(\theta)\left|T_{i}^{\vec{\alpha}}(\theta)\right|^{2}\right]}{d \theta} .
$$

In order to evaluate these expressions further, we need to know explicitly the precise form of the transmission matrix, i.e. the concrete form of the defect. An interesting situation occurs when the defect is transparent or rapidity independent, that is $\left|T_{i}^{\vec{\alpha}}(\theta)\right| \rightarrow\left|T_{i}^{\vec{\alpha}}\right|$, in which case we can pursue the analysis further. Noting that $d \epsilon(\theta) / d \theta=2 \delta(\theta)$, we obtain

$$
\left.\lim _{r \rightarrow 0} G_{i}^{\vec{\alpha}}(r) \sim \frac{q_{i}}{\pi r} \frac{\left|T_{i}^{\vec{\alpha}}\right|^{2}}{1+\exp \varepsilon_{i}(0, r, 0)} \frac{d \varepsilon_{i}(0, r, V / 2)}{d V}\right|_{V=0} .
$$

The derivative $d \varepsilon_{i}(0, r, V / 2) / d V$ can be obtained by solving recursively

$$
\frac{d \varepsilon_{i}(0, r, V / 2)}{d V}=-\frac{r}{2}-\sum_{j} N_{i j} \frac{1}{\left.1+\exp \varepsilon_{j}(0, r, V / 2)\right]} \frac{d \varepsilon_{j}(0, r, V / 2)}{d V},
$$

which results form a computation similar to a standard one in this context [ị̂] leading to the so-called constant TBA-equations. Here only the asymptotic phases of the scattering matrix enter via $N_{i j}=\lim _{\theta \rightarrow \infty}\left[\ln \left[S_{i j}(-\theta) / S_{i j}(\theta)\right]\right] / 2 \pi i$. The values of $\varepsilon_{i}(0, r, 0)$ needed in $\left(\overline{2} 2 . \overline{9}_{i}\right)$ can be obtained for small $r$ in the usual way from the standard constant TBAequations.

\subsection{Free Fermion with defects}

Let us exemplify the general formulae once more with the free Fermion. First of all we note that in this case in the TBA-equations (1.18i) the kernel $\varphi_{i j}$ is vanishing and the equation is simply solved by

$$
\varepsilon_{i}\left(\theta, r, \mu_{i}\right)=r m_{i} \cosh \theta-r \mu_{i} .
$$

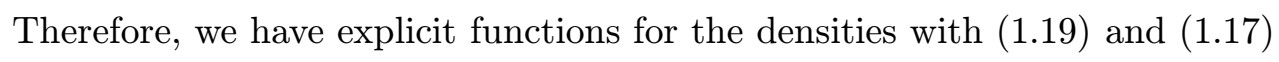

$$
\rho_{i}\left(\theta, r, \mu_{i}\right)=\frac{1}{2 \pi} m_{i} \cosh \theta \quad \text { and } \quad \rho_{i}^{r}\left(\theta, r, \mu_{i}\right)=\frac{m_{i} \cosh \theta / 2 \pi}{1+\exp \left(r m_{i} \cosh \theta-r \mu_{i}\right)} .
$$

According to $\left(\overline{2}, \overline{1} \overline{1}_{j}\right)$ the direct current reads

$$
\begin{aligned}
I^{\vec{\alpha}}(r, V)=\frac{q_{i}}{2} \int_{-\infty}^{\infty} d \theta & {\left[\rho_{\bar{\imath}}^{r}(\theta, r, V / 2)\left|T_{\bar{\imath}}^{\vec{\alpha}}(\theta)\right|^{2}-\rho_{i}^{r}(\theta, r,-V / 2)\left|T_{i}^{\vec{\alpha}}(\theta)\right|^{2}\right.} \\
& \left.\quad-\rho_{\bar{\imath}}^{r}(\theta, r,-V / 2)\left|\tilde{T}_{\bar{\imath}}^{\vec{\alpha}}(\theta)\right|^{2}+\rho_{i}^{r}(\theta, r, V / 2)\left|\tilde{T}_{i}^{\vec{\alpha}}(\theta)\right|^{2}\right] .
\end{aligned}
$$


Using atomic units $m_{e}=e=\hbar=m_{i}=q_{i}=1$, we obtain explicitly with (2.12i $)$

$$
I^{\vec{\alpha}}(r, V)=\frac{1}{\pi} \int_{0}^{\infty} d \theta \frac{\cosh \theta \sinh (r V / 2)\left|T^{\vec{\alpha}}(\theta)\right|^{2}}{\cosh (r \cosh \theta)+\cosh (r V / 2)},
$$

for $\left|T_{\bar{\imath}}^{\alpha}(\theta)\right|=\left|T_{i}^{\vec{\alpha}}(\theta)\right|=\left|\tilde{T}_{\bar{\imath}}^{\vec{\alpha}}(\theta)\right|=\left|\tilde{T}_{i}^{\alpha}(\theta)\right|=\left|T^{\vec{\alpha}}(\theta)\right|$. Then by $(\underset{2}{2} \cdot \vec{i})$ the conductance results to

$$
G^{\vec{\alpha}}(r)=r m \frac{e^{2}}{h} \int_{0}^{\infty} d \theta \frac{\cosh \theta\left|T^{\vec{\alpha}}(\theta)\right|^{2}}{1+\cosh (r m \cosh \theta)}
$$

in this case. We have re-introduced dimensional quantities instead of atomic units to be able to match with some standard results from the literature. The most characteristic features can actually be captured when we carry out the massless limit as indicated in section 2.3.2, which can be done even analytically. Substituting $t=e^{\theta}$, we obtain

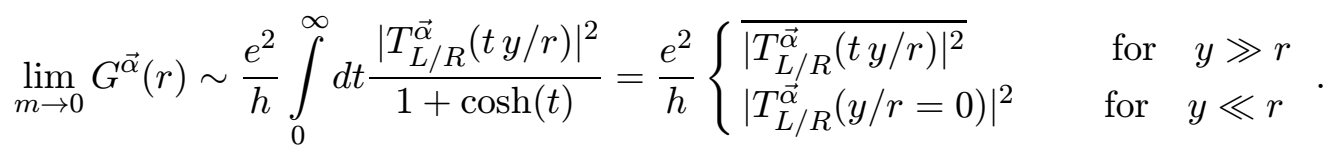

We have identified here two distinct regions. When $y \ll r$ we can replace the left/right transmission amplitudes by their values at $y / r=0$. When $y \gg r$ the transmission amplitudes enter the expression as a strongly oscillatory function in which $y / r$ plays the role of the frequency. It is then a good approximation to replace this function by its mean value as indicated by the overbar. It is straightforward to extend the expression (2.16) to the case when the assumption on $T^{\alpha}$ in (2.14) is relaxed and to the case with different values of $y$. To proceed further we need to specify the defect.

\subsubsection{Transparent defects, $\left|T^{\vec{\alpha}}\right|=1$}

Let us first consider the easiest example, which supports the general working of the method. When the defect is transparent, i.e., $\left|T^{\vec{\alpha}}\right|=1$, we can compute the expression for the conductance $(2.15)$ directly in the large temperature limit and obtain the well known behaviour $[\overline{2} \overline{4} \overline{4}]$

$$
\lim _{r \rightarrow 0,\left|T^{\vec{\alpha}}\right| \rightarrow 1} G^{\vec{\alpha}}(r) \sim \frac{e^{2}}{h}\left(1-\frac{r m}{2}\right) .
$$

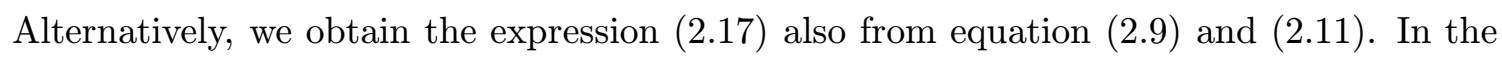
massless limit of $(2.16 i)$ we obtain $e^{2} / h$ which coincides with the result in [1] $\left.{ }^{1} \overline{1}\right]$. However, we should stress that we consider here purely massive cases and the massless limit only serves as a benchmark. Note that a transparent defect in this context does not necessarily mean the absence of the defect, since the transmission amplitude could be a non-trivial phase.

\subsubsection{The energy operator defect $\mathcal{D}^{\alpha}(\bar{\psi}, \psi)=g \bar{\psi} \psi$}

For this defect the computation of the conductance according to (2.15i) is more involved. The results of our numerical analysis of the expression (2.15i) are depicted in figure 3.

We observe several distinct features. First of all it is naturally to be expected that when we increase the number of defects the resistance will grow. This is confirmed, as for fixed 


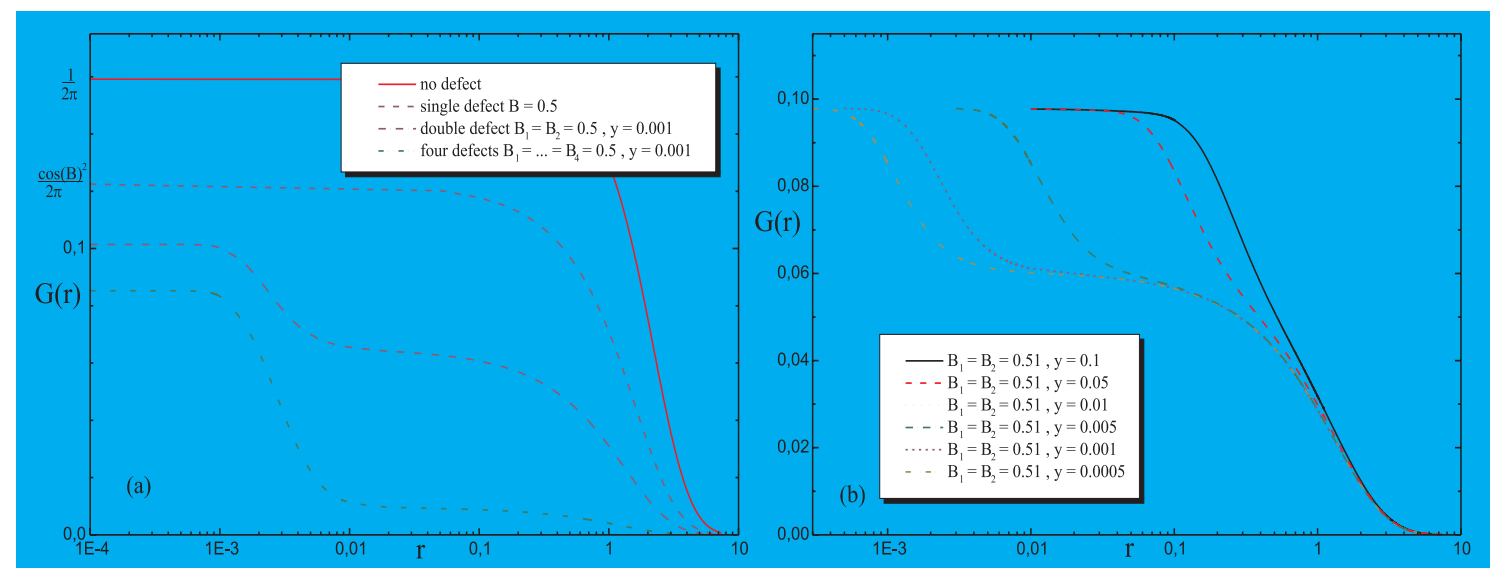

Figure 3: Conductance $G(r)$ for the complex free Fermion with the energy operator defects as a function of the inverse temperature $r$, for fixed effective coupling constant $B$ and (a) for varying amounts of defects $\ell=0,1,2,4$. (b) for $\ell=2$ for varying distances $y$.

temperature and increasing number of defects, the conductance decreases. Second we see several well extended plateaux. They can be reproduced with the analytical expressions obtained in the massless limit $(2 . \overline{1} \overline{6})$. To be able to compare with (2.15) we re-introduce atomic units for convenience, i.e. $e^{2} / h \rightarrow 1 / 2 \pi$. For a single defect there is only one plateau and from $(2.16 i)$ and the explicit expression for $T^{\alpha}(\theta)$ given in part I

$$
G^{\alpha}(r) \sim \frac{\cos ^{2} B}{2 \pi} .
$$

For $B=0.5$ the value 0.1226 is well reproduced in figure 3(a). The lower lying plateaux correspond to the region when $y \ll r$. In that case we obtain from $(2.16)$ together with the expressions for the reflection and transmission amplitudes of the double and four defect systems derived in part I

$$
\begin{aligned}
G^{\alpha_{1} \alpha_{2}}(r) & \sim \frac{1}{2 \pi}\left(\frac{\cos ^{2} B}{1+\sin ^{2} B}\right)^{2} & \text { for } \quad y \ll r \\
G^{\alpha_{1} \alpha_{2} \alpha_{3} \alpha_{4}}(r) & \sim \frac{1}{2 \pi}\left(\frac{\cos ^{4} B}{\cos ^{4} B-2\left(1+\sin ^{2} B\right)^{2}}\right)^{2} & \text { for } \quad y \ll r .
\end{aligned}
$$

For $B=0.5$ the values 0.0624 and 0.0095 are well reproduced in figure 3(a) for $\ell=2$ and $\ell=4$, respectively. The plateaux extending to the ultraviolet regime result from $(2.16 i)$ and by taking mean values of the expressions for the reflection and transmission amplitudes of the double and four defect systems given in part I

$$
\begin{aligned}
& G^{\alpha_{1} \alpha_{2}}(r) \sim \frac{2}{\pi} \frac{1+\sin ^{4} B}{\left(\cos ^{2}(2 B)-3\right)^{2}}, \quad \text { for } \quad y \gg r, \\
& G^{\alpha_{1} \alpha_{2} \alpha_{3} \alpha_{4}}(r) \sim \frac{1}{4 \pi}+\frac{\cos ^{8} B}{4 \pi\left[\cos ^{4} B-2\left(1+\sin ^{2} B\right)^{2}\right]^{2}}, \quad \text { for } \quad y \gg r .
\end{aligned}
$$

Also in this case the values for $B=0.5$, i.e., 0.110784 and 0.084311 for $\ell=2$ and $\ell=4$, respectively, match very well with the numerical analysis. Finally we have to explain the 
reason for the increase from one to the next plateaux and why the curves are shifted precisely in the way as indicated in figure 3(b) when we change the distance between the defects. This phenomenon is attributed to resonances, namely the existence of very sharp picks in the probability of transmission for two or more defects (see figure 1 in part I).

If we now compare the expressions (2.18)-(2.25i) with equations (3.34; part I)-(3.42; part I), we find complete agreement. This observation constitutes the central result of this work. We showed for a concrete integrable theory that the conductance computed by means

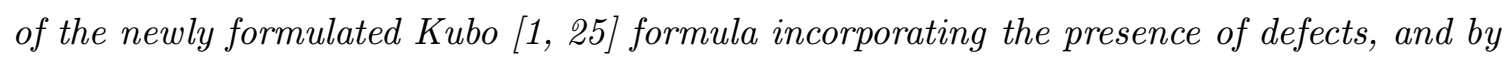
means of Landauer [i] formula are in perfect agreement. More concrete examples of this agreement are provided in [i],

\subsubsection{The $S U(3)_{2}$ homogeneous sine-Gordon model, unstable particles}

The $S U(3)_{2}$ homogeneous sine-Gordon (HSG) model is the simplest of its kind and contains only two self-conjugate solitons, which we denote by "+", "-", and one unstable particle, which we call $\tilde{c}$. The corresponding scattering matrix was found [i2 $\overline{2}]$ ] to be

$$
S_{ \pm \pm}=-1, \quad S_{ \pm \mp}(\theta)= \pm \tanh \frac{1}{2}\left(\theta \pm \sigma-\frac{i \pi}{2}\right)
$$

which means the resonance pole associated to the formation $\tilde{c}$ is situated at $\theta_{R}=\mp \sigma-i \pi / 2$, $\sigma$ being a free parameter. Stable bound states may not be formed. Since only for $S= \pm 1$ simultaneous reflection and transmission can occur [0.5], the $S U(3)_{2}$-HSG model only admits the presence of purely reflecting or transmitting defects. For the purely reflecting case, the expression (2.1.) vanishes so that the only non-trivial situation we can consider is a transparent defect, i.e. $|T|=1$. The results for the conductance after solving numerically

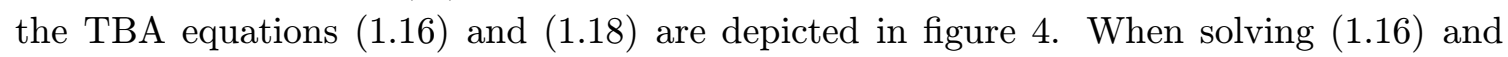

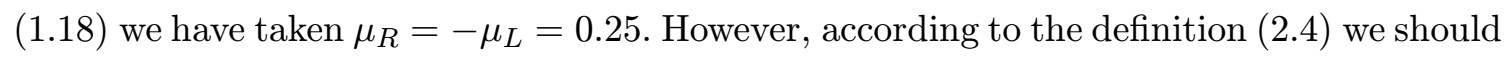
really consider the limit $\left(\mu_{R}-\mu_{L}\right) \rightarrow 0$. The reason why we instead take $\mu_{R}-\mu_{L}=0.5$ is that for this model we can of course not solve the TBA-equations analytically, as for the free Fermion. On the contrary, the numerics become fairly involved and they do not allow for considering the extreme limit $\left(\mu_{R}-\mu_{L}\right) \rightarrow 0$. However, we convinced ourselves that the results depicted in figure 4 reproduce indeed the correct behaviour of the conductance, since computing $G(r)$ in the deep ultraviolet limit for different values of $\mu_{R}-\mu_{L}$ leads always to the same plateau structure. We observe a relatively sharp increase in $G$ for an energy scale $2 \log r / 2 \sim-\sigma$ which corresponds to the onset of the unstable particle. In other words, only when a certain energy scale necessary for the excitation of the unstable particle is reached, the latter is formed and participates in the conducting process. All this information is encoded in the density $\rho_{i}^{r}\left(\theta, r, \mu_{i}\right)$. Computing now $\varepsilon_{i}(\theta, 0,0)$ in a standard TBA fashion we predict the plateaux from $(2.9)$ analytically at $1 / 2 \pi$ and $2(1+\sqrt{5}) /(5+$ $\sqrt{5}) \pi$. The last plateau corresponds to the deep ultraviolet limit, whereas the plateau at $1 / 2 \pi$ coincides with the value (2.17i) for a free Fermion theory when taking $e / h=1 / 2 \pi$. The reason is that the second plateau in figure 5 develops in the region when $\sigma \gg-2 \log r / 2$, that is $\sigma$ very large. In that limit we have $\lim _{\sigma \rightarrow \infty} S_{ \pm \mp}(\theta)=1$, such that the model becomes a free Fermion theory. 


\subsubsection{Resonances versus unstable particles}

In the light of the results of subsections 2.2.2 and 2.2.3 we can draw the conclusion that resonances in a double defect system and the presence of unstable particles may be described similarly [i] and 4 , it is clear that the plateau structures encountered do not differ much from each other. In particular, it seems that the parameter $y$ in the double defect system and the resonance parameter $\sigma$ in the $S U(3)_{2}$-HSG model play

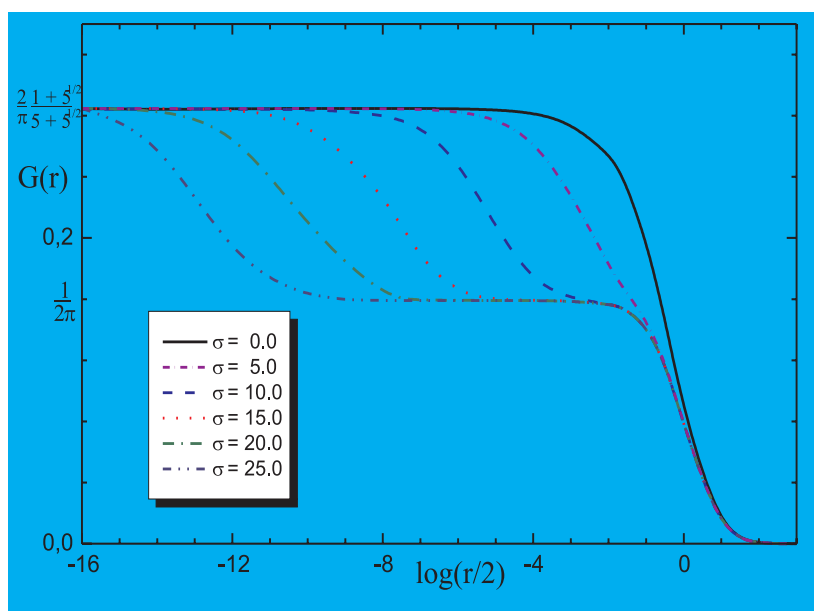

Figure 4: Conductance for the $S U(3)_{2}$-HSG-model. similar roles. Let us investigate more precisely these similarities, which from an intuitive point of view appear rather natural.

In the context of theories possessing unstable particle in their spectra, a very clear picture which explains the relatively sharp onset of the conductance with increasing temperature can be provided. The temperature at which this onset occurs, say $T_{C}$ can be related directly to the energy scale at which the unstable particle is formed, since then it starts to participate in the conducting process. The Breit-Wigner formula [i2 $\left.{ }_{-}^{2} \overline{6}\right]$ provides in this case the expressions for the mass $M_{\tilde{c}}$ and the decay width $\Gamma_{\tilde{c}}$ of the unstable particle $\tilde{c}$. Supposing that the particle $\tilde{c}$ is formed in the scattering process between particles of types $i$ and $j$ of masses $m_{i}, m_{j}$, this is reflected by a pole in $S_{i j}(\theta)$ at $\theta_{R}=\sigma-i \bar{\sigma}$. Setting $\bar{\sigma}=\pi / 2$, as corresponds to the model at hand, the Breit-Wigner formula for large values of the resonance parameter $\sigma$ gives

$$
M_{\tilde{c}} \approx \frac{1}{\sqrt{2}} \sqrt{m_{i} m_{j}} \exp |\sigma| / 2 \quad \text { and } \quad \Gamma_{\tilde{c}} \approx \sqrt{2 m_{i} m_{j}} \exp |\sigma| / 2 .
$$

Since a renormalization group flow is provided by mapping $M \rightarrow r M$, one observes that the quantity $M_{\tilde{c}}(r, \sigma)=r M=r e^{\sigma / 2}$ should remain invariant under the renormalization group flow. That means that if $r_{1}$ is the onset energy for the unstable particle $\tilde{c}$ for $\sigma=\sigma_{1}$ and $r_{2}$ is the onset energy for $\sigma=\sigma_{2}$, the conductance must satisfy the following scaling law

$$
G\left(r_{1}, \sigma_{1}\right)=G\left(r_{2}, \sigma_{2}\right) \quad \text { for } \quad r_{1} e^{\sigma_{1} / 2}=r_{2} e^{\sigma_{2} / 2} .
$$

This means we can control the position of the onset in the conductance by $M_{\tilde{c}}(r, \sigma)$.

Analyzing now the scaling behaviour of the conductance for the double defect system studied in subsection 2.2 .2 we find

$$
G\left(r_{1}, y_{1}\right)=G\left(r_{2}, y_{2}\right) \quad \text { for } \quad \frac{r_{1}}{y_{1}}=\frac{r_{2}}{y_{2}},
$$

Then the comparison with $(2.25 i)$ suggests that we can formally relate the distance between the two defects to the resonance parameter as $\sigma=2 \ln ($ const $/ y)$. However, despite the fact 
that the net result with regard to the conductance is the same, the origin of the onset is different. Whereas for the HSG-model it resulted from a change in the density distribution function $\rho_{i}^{r}\left(\theta, r, \mu_{i}\right)$ it is now triggered by the structure of $\left|T^{\alpha}(\theta)\right|$. Since for the free Fermion the function $\rho_{i}^{r}$ keeps its overall shape and just translates as the temperature is changed, the onset of the conductance occurs when the maxima of $\left|T^{\alpha}(\theta)\right|$ are reached. By analyzing the concrete expression of $T^{\alpha}(\theta)$ for the energy operator defect (see part I) it is easy to verify that for a double defect

$$
T^{\alpha_{1} \alpha_{2}}\left(\theta=\ln \left[\frac{(2 n+1) \pi}{y}\right]\right) \approx 1 \quad \text { for } \quad n \in \mathbb{Z} .
$$

Drawing an analogy to the scattering matrix of the HSG-model the values of $\theta$ for which $T^{\alpha_{1} \alpha_{2}}(\theta)$ is maximal play the same role as the value $\theta_{R}=\sigma-i \pi / 2$ corresponding to the resonance pole of the S-matrix. In that sense we can make the identification $\sigma_{n}=$ $\ln [(2 n+1) \pi / y]$. There are however some differences between both systems, since in the case of the $S U(3)_{2}$-HSG model the onset of the conductance is due to a single unstable particle, whereas for the double defect system the same effect can be attributed to several maxima of the transmission probability. The other important difference is that $y$ is now a measurable quantity, so that the "mass" of the resonances can be experimentally accessible.

\subsubsection{Multiple plateaux}

Up to now, we have observed that we always obtain essentially two plateaux in the conductance, no matter how many $(\geq 2)$ and what type of defects we implement. The natural question arising at this point is whether it is possible to have a set up which leads to a more involved plateaux structure. It is clear that if we had many defects in a row separated far enough from each other such that the relaxation time of the passing particles is so large that they could be treated as single rather than multiple defects, then any desired type of multiple plateau structure could be obtained. In this case the conductance is simply the sum of the expressions one has for each defect independently. Recalling the origin of the different plateaux, there is another slightly less obvious option. The density distribution function $\rho^{r}$ is a peaked function of the rapidity

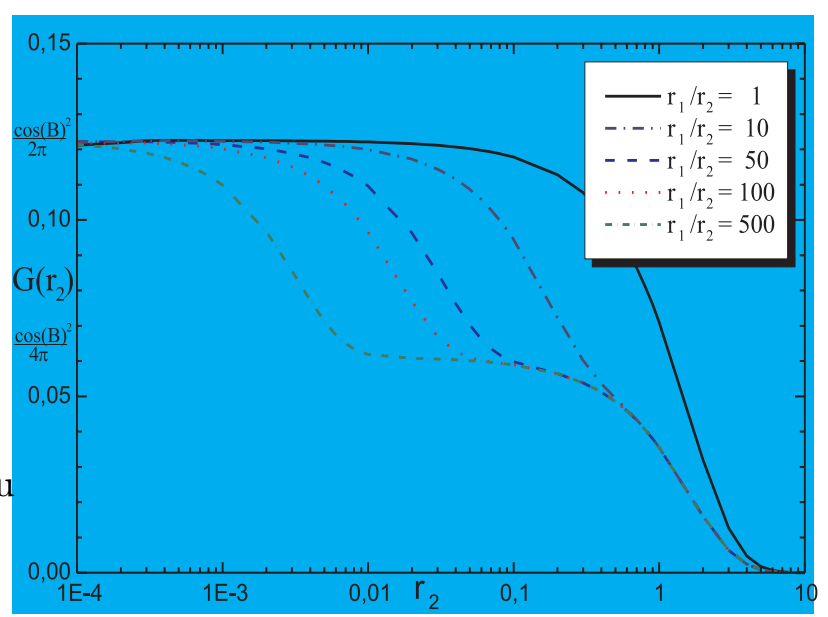

Figure 5: Conductance $G\left(r_{2}\right)$ for the complex free Fermion with the energy operator defects as a function of the inverse temperature $r_{2}$, for fixed effective coupling constant $B=0.5$ and varying temperature ratios in the two halves of the wire. and if the resonances in $T^{\alpha}(\theta)$ would be separated far enough, such that they are resolved by $\rho^{r}$, we would also get a multiple plateaux pattern. However, tuning the distance between the defects or the coupling constant will merely translate the position of the resonances in 
the rapidity variable or change their amplitudes, respectively (see section 2, part I). Therefore the last option left is to change the $\rho^{r} \mathrm{~s}$, which is possible by varying the temperature. Choosing now a configuration as in figure 2 with different temperatures $T_{1}$ and $T_{2}$, one can "create" a second plateau at half the height of the original one. The reason for this is simply that the cooled half of the wire will cease to contribute to the conductance as can be directly deduced from (2.15). We depict the results of our computations in figure 5. From this it also obvious that if we only cool the fraction $x$ of the wire, the lowest plateau will be positioned at the height $x$ times the height of the upper plateau. Thus, by combining these different configurations, i.e., different temperatures or defects, we could produce any desired plateau structure.

\section{Conclusions and open problems}

In this section I will present the main conclusions of my talk and also of part I, since the main aim of this work was actually to compare the two theoretical descriptions presented in the two parts. In our work we have exploited the special features of $1+1$ dimensional integrable quantum field theories in order to compute the DC conductance in an impurity system. For this purpose several non-perturbative techniques have been used. As the main tools we employed the thermodynamic Bethe ansatz in a Landauer transport theory computation and the form factor expansion in the Kubo formula.

The comparison between the Kubo formula (1.1; part I) and the Landauer formula (2..-1) yields in particular an identical plateau structure for the DC conductance in the ultraviolet limit.

We have explained to what extend integrability can be exploited in order to determine the reflection and transmission amplitudes through a defect. Unfortunately, for the most interesting situation in this context, namely when $R / \tilde{R}$ and $T / \tilde{T}$ are simultaneously nonvanishing, the Yang-Baxter bootstrap equations narrow down the possible bulk theories

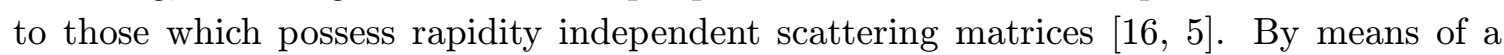
relativistic potential scattering theory we computed for several types of defects the $R / \tilde{R} \mathrm{~s}$ and $T / \tilde{T}_{\mathrm{s}}$, thus enlarging the set of examples available at present. We confirm that for real potentials parity is preserved, but otherwise essentially all possible combinations of parity breaking can occur. From the knowledge of the single defect amplitudes the multiple defect amplitudes, which exhibit the most interesting physical behaviours, can be computed in a

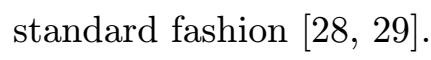

We have newly proposed a Kubo formula [25 5 , which accommodates the situation when defects are present (1.1; part I). We evaluated the current-current correlation functions occurring in there by means of a non-perturbative method based on integrability, namely

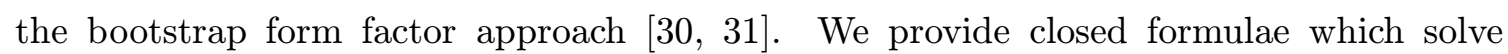
explicitly the defect recursive equations involving any arbitrary number of particles. We predict the plateaux in the conductance as a function of the temperature analytically.

We newly formulated the TBA equations for a defect with simultaneously non-vanishing reflection and transmission amplitudes. We indicate how these equations can be used to compute various thermodynamic quantities, which are, however, most interesting only 
when considered per unit length. By means of the TBA we compute the density distribu-

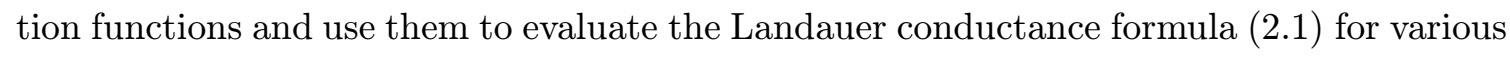
defects in a complex free Fermionic theory. Also in this case, we predict analytically the most prominent features in the conductance as a function of the temperature, i.e. the plateaux.

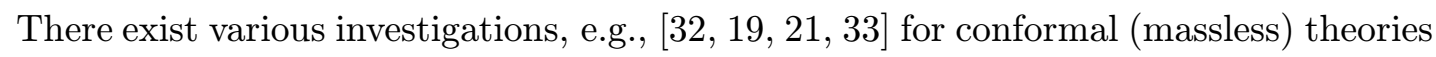
with defects, which exploit the original folding idea of Wong and Affleck [ije- The idea is that a conformal field theory with a purely transmitting or reflecting defect can be mapped into a boundary theory, i.e. a theory living in half space, which has the advantage that the full restriction of modular invariance can be exploited in the construction of

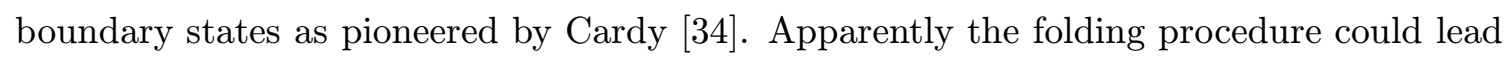
to non-trivial solutions for the reflection and transmission amplitudes starting with a purely reflecting or transmitting theory. However, one should stress that the folding is carried out on the basis of the field content of the conformal field theory, whereas our analysis is based on a particle description, namely we take the ZF-algebra as our starting point. Therefore, the transmission and reflection amplitudes obtained by means of the folding technique can not be compared with the objects we study here, even in the conformal limit.

In this context, there are several interesting open issues. Most challenging is to treat in full generality the massive and temperature dependent case of (1.1; part I). Unfortunately, the formulation of non-perturbative methods does not yet cover that situation [i6] $[\overline{6} \mid$ and it remains to be clarified how the form factor bootstrap program for the computation of twopoint functions can be extended to that case. It would be further interesting to compute thermodynamic quantities per unit length by means of the TBA and to develop methods for the systematic classification of integrable defects.

Proceeding further in our investigation of the applications of integrable models to the description of realistic physical systems, we have established that coupling an impurity in a quantum wire to an external monochromatic electromagnetic field leads to high harmonic generation [i $2 \overline{2}]$. Harmonic generation i.e., the emission of multiples of the incoming frequency when a system is coupled to a monochromatic field, has been widely studied in the context of atomic physics. However, up to now there were no results for solid state materials. The concrete system we have studied is a quantum wire described by means of the Dirac equation doped with a defect which couples minimally to an external field of frequency $\omega$. Considering separately the situations corresponding to a single and a double defect system we observed that, for the particular type of defect treated, only even multiples of the incoming frequency are emitted for the single defect, whereas all even and odd multiples are generated for the double defect system. These features are observed both in the Fourier expansion of the transmission probability through the defect and in the emission spectrum of the dipole momentum. It would be extremely interesting to confirm our findings experimentally.

\section{Acknowledgments}

We would like to thank the organizers of this workshop for the opportunity to present these 
talks, for financial support and for making the celebration of the $50^{\text {th }}$ anniversary of the Instituto de Física Teórica (IFT) a very enjoyable and rewarding event. In addition, we would like to thank Carla Figueira de Morisson Faria (Max Born Institut Berlin) and Frank Göhmann (Universität Bayreuth) for their participation in [2] also grateful to the Deutsche Forschungsgemeinschaft (Sfb288) for financial support.

\section{References}

[1] O.A. Castro-Alvaredo and A. Fring, From integrability to conductance, impurity systems, hep-th/0205076.

[2] O.A. Castro-Alvaredo, A. Fring and C. Figueira de Morisson Faria, Relativistic treatment of harmonics from impurity systems in quantum wires, cond-mat/0208128.

[3] O.A. Castro-Alvaredo and A. Fring, Nucl. Phys. B618 [FS] (2001) 437.

[4] O.A. Castro-Alvaredo and A. Fring, Unstable particles versus resonances in impurity systems, conductance in quantum wires, cond-mat/0112199.

[5] O.A. Castro-Alvaredo, A. Fring and F. Göhmann, On the absence of simultaneous reflection and transmission in integrable impurity systems, hep-th/0201142.

[6] O.A. Castro-Alvaredo and A. Fring, Nucl. Phys. B636 (2002) 611.

[7] R. Landauer, IBM J. Res. Dev. 1 (1957) 223; Philos. Mag. 21 (1970) 863 ; M. Büttinger, Phys. Rev. Lett. 57 (1986) 1761.

[8] C.N. Yang and C.P. Yang, Phys. Rev. 147 (1966) 303; J. Math. Phys. 10 (1969) 1115.

[9] Al.B. Zamolodchikov, Nucl. Phys. B342 (1990) 695.

[10] M.J. Martins, Nucl. Phys. B426 [FS] (1994) 661.

[11] A. LeClair, G. Mussardo, H. Saleur and S. Skorik, Nucl. Phys. B453 (1995) 581.

[12] A.B. Zamolodchikov and Al.B. Zamolodchikov, Ann. of Phys. 120 (1979) 253;

L.D. Faddeev, Sov. Sci. Rev. Math. Phys. C1 (1980) 107.

[13] I.V. Cherednik, Theor. Math. Phys. 61 (1984) 977.

[14] E.K. Sklyanin, J. Math. Phys. A21 (1988) 2375.

[15] A. Fring and R. Köberle, Nucl. Phys. B421 (1994) 159; Nucl. Phys. B419 [FS] (1994) 647; Int. J. of Mod. Phys. A10 (1995) 739.

[16] G. Delfino, G. Mussardo and P. Simonetti, Phys. Lett. B328 (1994) 123, Nucl. Phys. B432 (1994) 518.

[17] O.A. Castro-Alvaredo, A. Fring, C. Korff and J.L. Miramontes, Nucl. Phys. B575 (2000) 535.

[18] T.R. Klassen and E. Melzer, Nucl. Phys. B338 (1990) 485; Nucl. Phys. B350 (1991) 635; Nucl. Phys. B370 (1992) 511.

[19] P. Fendley, A.W.W. Ludwig and H. Saleur, Phys. Rev. B52 (1995) 8934.

[20] F. Lesage, H. Saleur and S. Skorik, Nucl. Phys. B474 (1996) 602.

[21] P. Fendley, A.W.W. Ludwig and H. Saleur, Phys. Rev. Lett. 74 (1995) 3005.

[22] J.-S. Caux, H. Saleur and F. Siano, Phys. Rev. Lett. 88 (2002) 106402. 
[23] S. Ghoshal and A.B. Zamolodchikov, Int. J. of Mod. Phys. A9 (1994) 3841.

[24] C.L. Kane and M.P.A. Fisher, Phys. Rev. B46 (1992) 15233.

[25] R. Kubo, Can. J. Phys. 34 (1956) 1274.

[26] G. Breit and E.P. Wigner, Phys. Rev. 49519 (1936).

[27] J.L. Miramontes and C.F. Fernández-Pousa, Phys. Lett. B472 (2000) 392.

[28] C. Cohen-Tannoudji, Quantum Mechanic, (John Wiley \& Sons, New York, 1977).

[29] E. Merzbacher, Quantum Mechanic, (John Wiley \& Sons, New York, 1970).

[30] P. Weisz, Phys. Lett. B67 (1977) 179; M. Karowski and P. Weisz, Nucl. Phys. B139 (1978) 445.

[31] F.A. Smirnov, Form Factors in Completely Integrable Models of Quantum Field Theory, Advanced Series in Mathematical Physics, Vol. 14, World Scientific, Singapore, 1992.

[32] E. Wong and I. Affleck, Nucl. Phys. B417 (1994) 403.

[33] M. Oshikawa and I. Affleck, Nucl. Phys. B495 (1997) 533; A. LeClair, A.W.W. Ludwig and G. Mussardo, Nucl. Phys. B512 (1998) 523; A. LeClair and A.W.W. Ludwig, Nucl. Phys. B549 (1999) 546; C. Nayak, M.P.A. Fisher, A.W.W. Ludwig and H.H. Lin, Phys. Rev. B59 (1999) 15694; T. Quella and V. Schomerus, Symmetry breaking boundary states and defect lines, hep-th/0203161.

[34] J.L. Cardy, Nucl. Phys. B324 (1989) 581. 